\title{
Bewegungsgesundheit der Skapula
}

\author{
Thomas Karlinger
}

Bewegungsgesundheit bedeutet, viel Bewegungsvariabilität zur Verfügung zu haben. Therapeuten untersuchen die Bewegungsgesundheit, indem sie das bevorzugte Bewegungsmuster und die kognitive Bewegungskontrolle analysieren. Fundierte anatomische Kenntnisse und eine scharfe Beobachtungsgabe sind dabei wesentlich, um das Problem zu identifizieren.

\section{Anwendung des Kinetic Control Frameworks}

Die Kinetic Control Gruppe um Sarah Mottram und Mark Comerford definierte den Begriff der Bewegungsgesundheit [1]. Bewegungsgesundheit beschreibt demnach einen Zustand, in dem eine Person nicht nur verletzungsfrei ist, sondern auch die Möglichkeit besitzt, zu wählen, wie sie eine Bewegung ausführt [2][3]. Wird dieser Begriff nun auf eine optimale Skapulafunktion übertragen, so stellt sich nicht mehr die Frage, ob es richtige oder falsche Bewegungen der Skapula gibt. Es stellt sich in Untersuchung und Behandlung vielmehr die Frage nach der Bewegungsvariabilität der Skapula.

\section{Verlorene Bewegungsmöglichkeiten}

Wie ein Klavierspieler auf seinem Instrument viele Tasten zur Verfügung hat, um eine Vielzahl an Melodien zu spielen, so sollte auch unser Bewegungsapparat ein großes Repertoire an Bewegungsmöglichkeiten besitzen, um einen Bewegungsauftrag durchzuführen. Studien über die Bewegungsvariabilität zeigen, dass eine eingeschränkte Variabilität der Bewegung in Zusammenhang mit Schmerzen steht [4][5]. Kommt es also zum Verlust von Bewegungsmöglichkeiten (weniger Tasten am „Bewegungsklavier“), so ist das Individuum gezwungen, sich weniger variabel zu bewegen, was wiederum zu einer häufigeren Belastung derselben Strukturen führt. Ob diese eingeschränkte Bewegungsvariabilität nun zu Symptomen führt, hängt von den Gegebenheiten der Umwelt, des Individuums und der Aufgabenstellungen ab, die auf das individuelle Bewegungssystem einwirken [6][7]. So ist es nicht nur ein einzelner Faktor, der das Risiko für Verletzungen erhöht, sondern vielmehr das Zusammenwirken dieser drei Einflussfaktoren. Møller et al. aus Dänemark konnten zum Beispiel 2017 in einer Kohortenstudie mit jungen Elite -Handballspielern zeigen, dass Skapuladysfunktionen bei einer
Erhöhung der Trainingsbelastung einen verstärkenden Faktor für das Verletzungsrisiko der Schulter darstellen [8].

\section{Untersuchung der Bewegungs- gesundheit}

Die Untersuchung der Bewegungsqualität stellt Therapeuten vor eine große Herausforderung, da sich jeder Mensch auf seine individuelle Art bewegt und Kategorien wie „Richtig oder Falsch“ hier nicht zielführend sind. Basierend auf einer Kombination aus aktueller wissenschaftlicher Literatur zum Thema Bewegungskontrolle, klinischer Erfahrung und einem umfassenden Clinical Reasoning Prozess schlagen Dingenen et al. ein Evaluationsmodell vor, das die individuelle Bewegungsvariabilität miteinbezieht [6]. Die Autoren unterscheiden zwischen der Untersuchung des bevorzugten Bewegungsmusters und der Untersuchung der kognitiven Bewegungskontrolle.

\section{Dingenen et al. schlagen die \\ Untersuchung des bevorzugten \\ Bewegungsmusters und der kogni- \\ tiven Bewegungskontrolle vor, um die Bewegungsgesundheit $z u$ evaluieren.}

\section{Untersuchung des bevorzugten (natürlichen) Bewegungsmusters}

Bei dieser Form der Bewegungsuntersuchung wird der Patient gebeten, einfache, funktionelle Bewegungen auszuführen, ohne spezifische Instruktionen zu erhalten. Die natürlichen Bewegungsmuster geben einen Hinweis darauf, wie der untersuchte Gelenkbereich im täglichen Leben oder während sportlicher Aktivitäten bewegt und belastet wird. 


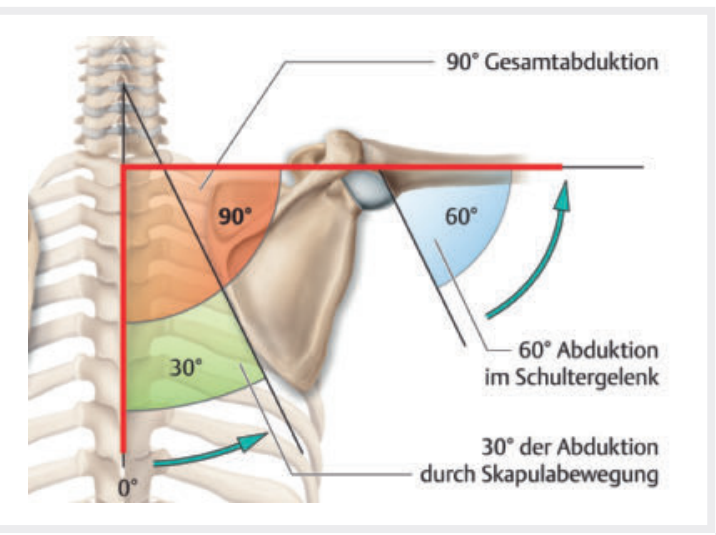

Abb. 1 Skapulohumeraler Rhythmus. (Quelle: Hochschild J. Strukturen und Funktionen begreifen, Funktionelle Anatomie. Band 1: Wirbelsäule und obere Extremität. 5. Aufl. Stuttgart: Thieme; 2019)

\section{Skapulohumeraler Rhythmus}

Um das natürliche Bewegungsmuster der Skapula zu untersuchen, eignet sich die natürliche, spontane Elevationsbewegung des Armes. Hier kommt die Analyse des skapulohumeralen Rhythmus ins Spiel, der sowohl statisch als auch dynamisch analysiert werden kann. Die Elevation des Armes setzt sich aus glenohumeraler Elevation, Aufwärtsrotation, posteriorem Tilt der Skapula und leichter Rumpfbewegung zusammen. Für je zwei Grad an glenohumeraler Elevation sollte die Skapula ein Grad mitbewegen (Rhythmus 2:1) ( Abb. 1).

Obwohl glenohumerale Bewegung und Skapulabewegung simultan ablaufen sollten, findet die Bewegungsinitiierung vor allem im Glenohumeralgelenk (GHG) statt (Abduktion bis ca. $60^{\circ}$ und Flexion bis ca. $90^{\circ}$, bis die Skapula mitbewegt). Bei endgradiger Elevation von $180^{\circ}$ sollte der Margo medialis der Skapula also in $60^{\circ}$ Aufwärtsrotation stehen. Als klinische Hilfe dient hier der Angulus inferior der Skapula, der sich in endgradiger Elevation durch die Aufwärtsrotation bis zur mittleren Axillarlinie vorbewegen, jedoch nicht mehr als 1,5 Zentimeter vom Brustkorb nach lateral abstehen sollte [1] ( $\mathbf{A} \mathbf{A b b} .2)$.

\section{Winging - Tilt}

Die Beobachtung des Skapula-Wingings oder des anterioren Tilts während der natürlichen Bewegung stellt eine Beeinträchtigung der skapulothorakalen Bewegungsfähigkeit dar, die lediglich Hinweise auf eine eingeschränkte Bewegungsgesundheit geben kann. Von eingeschränkter Bewegungsgesundheit ist dann die Rede, wenn der Patient keine Möglichkeit oder Fähigkeit besitzt, diese Beeinträchtigung zu korrigieren. Die Analyse und Interpretation des Skapula-Wingings oder anterioren Tilts steht in Zusammenhang mit der synergistischen und koordinatorischen Effizienz der globalen Stabilisatoren und globalen Mobilisatoren der Skapula. Darüber hinaus lassen sich jedoch auch noch andere Beeinträchtigungen der optimalen Ska-

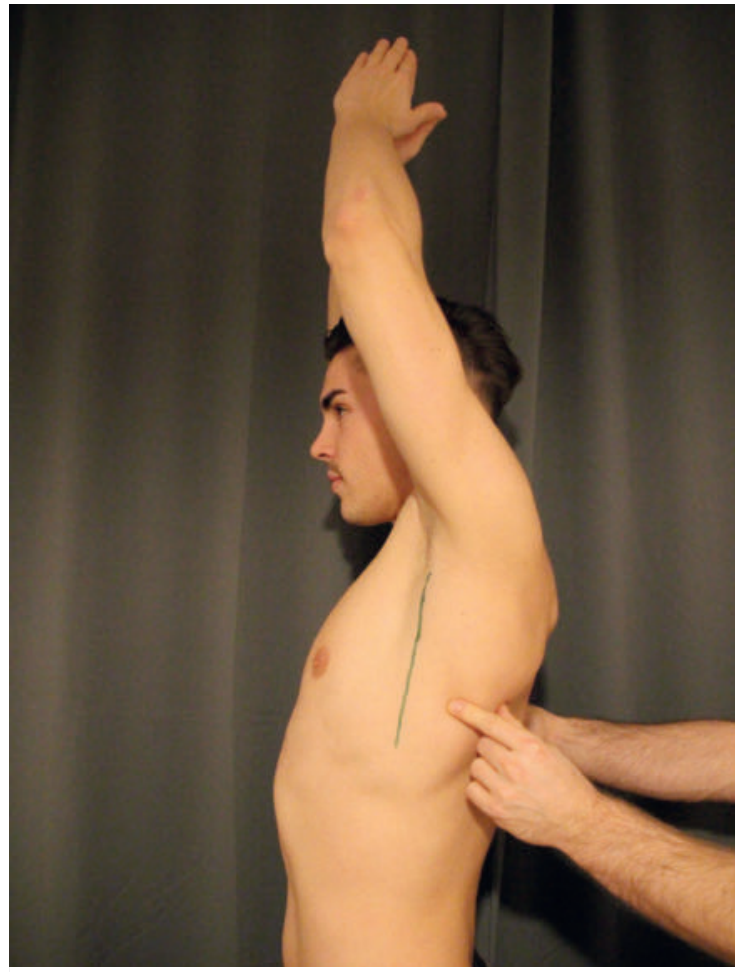

Abb. 2 Der Angulus inferior sollte sich bei endgradiger Elevation bis zur mittleren Axillarlinie bewegen (Symbolbild). (Quelle: T. Karlinger)

pulabewegung feststellen, wie zum Beispiel verminderte Aufwärtsrotation, vermehrte Elevation oder Protraktion, die ebenfalls durch fehlerhafte Muskelsynergien hervorgerufen werden.

So kann zum Beispiel ein Skapula-Winging während der konzentrischen Elevation mit einer schlechten Funktion des M. serratus anterior in Zusammenhang stehen, während hingegen das Auftreten des Wingings in der exzentrischen Phase der Elevation ein Timing-Problem darstellen kann, bei dem die skapulohumeralen Muskeln weniger schnell entspannen als die skapulothorakalen Muskeln [1]. Dieses fehlerhafte Aktivierungsmuster konnte bei Patienten mit subakromialem Impingement gemessen und durch gezieltes Retraining der Bewegungskontrolle wieder rückgängig gemacht werden. Ebenso führte diese Behandlung auch zur Verbesserung der Symptome [9].

\section{Untersuchung der kognitiven Bewegungs- kontrolle}

Die bloße Beobachtung, ob die Bewegungsfähigkeit beeinträchtigt ist, reicht nicht aus, um Aufschluss über die Fähigkeit der Bewegungskontrolle zu erhalten. Hierfür werden Tests der kognitiven Bewegungskontrolle verwendet. Diese Tests wurden in Studien an unterschiedlichen Körperregionen und unterschiedlichen Populationen eingesetzt und zeigten gute bis exzellente Inter- und Intra-Tester Reliabilität [10][11][12]. 
Diese Tests stellen keine funktionellen Tests dar. Sie überprüfen die Fähigkeit, die Freiheitsgrade des Körpers bewusst zu kontrollieren. Die Testperson wird vor die Herausforderung gestellt, eine Bewegung in einem Gelenk zu eliminieren, während ein darüber- oder darunterliegendes Gelenk in einer Ebene bewegt wird. Die Tests besitzen eine klar definierte Ausgangsstellung und eine klar definierte Standardgrenze (Benchmark), die erreicht werden muss. Während der Testung werden die verwendeten Bewegungsstrategien beobachtet und beurteilt [13]. Die Testperson wird gebeten, auftretende unkontrollierte Bewegungen zu verhindern, wodurch ein kognitives Element hinzugefügt wird. Diese Vorgehensweise gibt zum einen Aufschluss darüber, ob eine Bewegung bewusst kontrolliert werden kann, und informiert zum anderen über den Status der Bewegungsgesundheit der Testperson. Denn je mehr koordinative Strategien bewusst verwendet werden können, um ein Bewegungsergebnis zu erzielen, desto mehr Bewegungsoptionen stehen der Person zur Verfügung.

\section{Testung der kognitiven Kontrolle der Skapula}

Das Kinetic Control Framework beschreibt die Testung der kognitiven Bewegungskontrolle der Skapula. Jeder Test beginnt mit dem Positionieren der Skapula in die Neutralstellung (siehe Kasten Neutralstellung der Skapula) ( $>$ Abb. 3). Der Patient wird zuerst passiv aus seiner individuellen Ruheposition in die Neutralstellung geführt ( $\mathbf{A b b . 4 )}$ ), um dann im Anschluss diese Position aktiv (mit minimalem Kraftaufwand) zu halten [1][13].

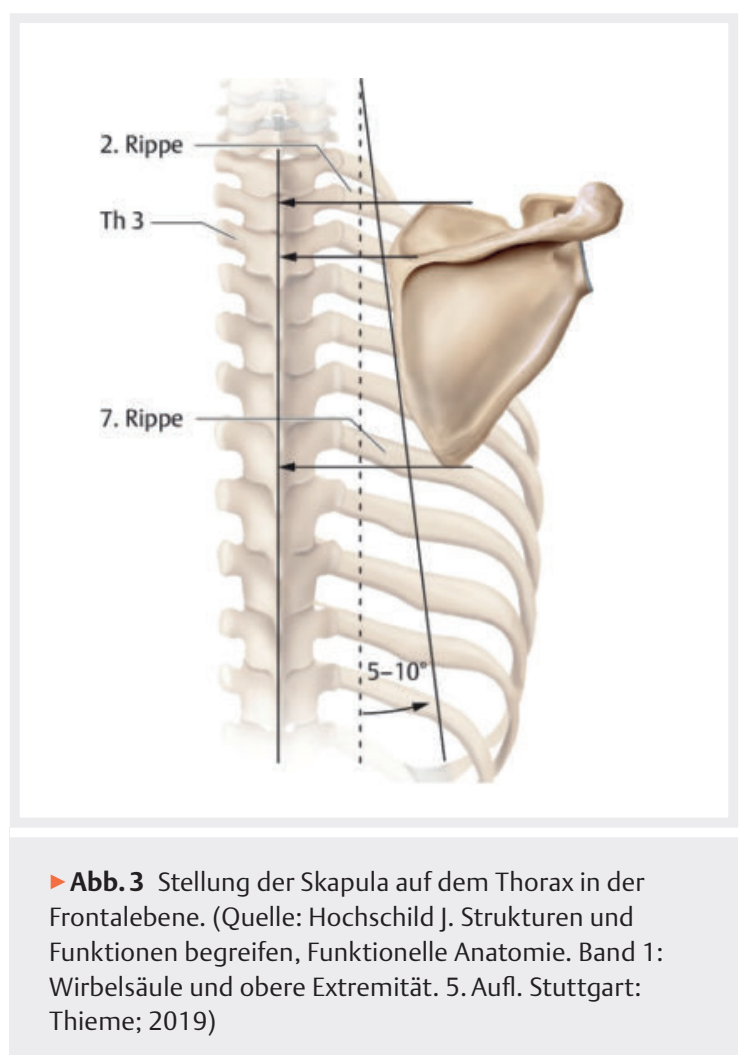

NEUTRALSTELLUNG DER SKAPULA ( ABB. 3)

- Angulus superior auf Höhe des Proc. spinosus T2

- mediale Spina scapulae auf Höhe des Proc. spinosus T3

- Akromion höher als Angulus superior

- Margo medialis \&Angulus inferior scapulae in Kontakt mit dem Brustkorb

Häufig befindet sich die Skapula in ihrer Ruhestellung in einer Position, in der das Glenoid nach inferior und anterior geneigt ist (IAG Position). Untersuchungen haben gezeigt, dass Haltungsabweichungen in Richtung Depression der Skapula in Zusammenhang mit einer gesteigerten neurodynamischen Mechanosensitivität und einer erhöhten lokalen Druckempfindlichkeit stehen [14]. Grund dafür könnten Veränderungen im Thoracic Outlet durch eine zu tiefe Klavikula sein, die zu vermehrter Spannung des Plexus brachialis und zu einer verminderten Bewegungsfreiheit der Mm. scaleni führt, da die erste Rippe durch die tiefstehende Klavikula nach inferior gedrückt wird. Eine mögliche Hypothese hinter der vermehrten lokalen Druckempfindlichkeit könnte auch eine veränderte Kraftverteilung der Lasten sein, die mit der oberen Extremität bewegt werden. Jede Gewichtsbelastung der oberen Extremität lastet auf der Skapula, welche die Aufgabe hat, diese Kräfte auf die gesamte Brustwirbelsäule (M. trapezius pars transversa und pars ascendens), Brustkorb (M. serratus) und Halswirbelsäule (M. trapezius pars descendens) zu verteilen.

Obwohl aufgrund von Haltungsabweichungen nicht auf Symptome geschlossen werden kann, erhalten wir im klinischen Alltag durch die Untersuchung der Ruheposition der Skapula viele Hinweise auf veränderte Muskellängen, die wiederum unser weiteres Vorgehen bestimmen. Die IAG Position wird hervorgerufen durch vermehrte Aktivität der Abwärtsrotatoren der Skapula (globale Mobilisatoren) und verminderte Aktivität der Aufwärtsrotatoren (globale Stabilisatoren) ( Tab. 1). Die passive Korrektur der Skapula in Richtung Neutralstellung zeigte in einer Untersuchung deutliche Verbesserungen der Schmerzen, der Propriozeption und des Bewegungsausmaßes [15].

Ausgehend von der Neutralposition erhält der Patient den Bewegungsauftrag, den Arm bis in eine Höhe von $90^{\circ} \mathrm{zu}$ flektieren (oder $60^{\circ}$ zu abduzieren), ohne dabei die Neutralposition der Skapula zu verlieren. Der Therapeut beob-

-Tab. 1 Muskulatur der Skapula

\begin{tabular}{|l|l|}
\hline Globale Stabilisatoren & Globale Mobilisatoren \\
\hline \begin{tabular}{l|l} 
- M. serratus anterior & - M. levator scapulae \\
- M. trapezius pars as- & - Mm. rhomboidei \\
cendens/pars transversa & - M. pectoralis minor
\end{tabular} \\
\hline
\end{tabular}


achtet eventuell auftretende Bewegungen der Skapula und weist den Patienten darauf hin, diese zu verhindern. Kann der Patient diese Bewegungen nicht verhindern oder die Standardgrenze nicht mehr erreichen, so kann von einer eingeschränkten Bewegungskontrolle der Skapula ausgegangen werden.

Benannt wird die Bewegungskontrollstörung nach der Richtung der auftretenden unkontrollierten Bewegung. Durch diese Analyse kann eine Bewegungsdiagnose formuliert werden. Bewegt sich die Skapula während der Armflexion zum Beispiel in Richtung Abwärtsrotation und kann diese vom Patienten nicht aktiv verhindert werden, spricht man von unkontrollierter Skapula Abwärtsrotation (= Bewegungsdiagnose). Häufig klinisch zu beobachtende unkontrollierte Bewegungen bei Abduktion/Flexion sind [1][7]:

- Abwärtsrotation

- Winging

- Anteriorer Tilt

- Elevation

\section{Individuelles Retraining der} Bewegungsgesundheit

Der beschriebene Test untersucht die koordinatorische Effizienz und führt zur Formulierung einer Bewegungsdiagnose [7][16]. Die Anwendung des Kinetic Control Frameworks wird wesentlich durch diese Diagnose bestimmt, da der Therapeut sein weiteres Vorgehen in Untersuchung und Behandlung darauf abstimmt [1]. Eine unkontrollierte Skapula Abwärtsrotation erfordert weitere Untersuchung der Kontraktionseffizienz des M. serratus anterior und M. trapezius pars ascendens (Aufwärtsrotatoren) sowie eine Untersuchung der Muskeldehnfähigkeit von M. levator scapulae, Mm. rhomboidei, M. pectoralis minor und anderen Abwärtsrotatoren der Skapula. Die Behandlung dieser Synergien und die damit verbundene Verbesserung der Bewegungsgesundheit führen zu einer individuellen Problem-Management-Strategie, die auf die vorhandenen Einschränkungen eingeht.

\section{Retraining der Effizienz}

Qualität vor Quantität: Der menschliche Körper ist darauf programmiert, seine Bewegungsziele zwar effektiv, nicht jedoch effizient zu erreichen. Während die effektive Bewegung lediglich den Fokus auf das Ziel legt (zum Beispiel Arm-Elevation bis über Kopf), so steht bei einer effizienten Bewegung auch der Weg zu diesem Ziel im Vordergrund. Effiziente Bewegung und Haltung der Skapula ermöglichen dem Glenohumeralgelenk und dem Nacken, sich kontrolliert zu bewegen, ohne dass Scherbelastungen entstehen. Das Retraining der koordinatorischen Effizienz zielt darauf ab, die Balance zwischen globalen Stabilisatoren und globalen Mobilisatoren des jeweiligen Körperbereichs wiederherzustellen. Diese Dysbalancen können sich sowohl bei niedrigschwelligen (low threshold) Aktivitäten als auch bei hochschwelligen (high threshold) Aktivitäten zeigen [13]. Das Retraining muss auch in Bezug auf die Aktivierungsschwellen angepasst werden.

\section{Retraining der Skapula Orientierung und Koordination}

Um die Balance der Synergisten wiederherzustellen, sollte zunächst die optimale Skapula Orientierung (Neutralstellung) eingeübt werden. Dies sollte zuerst passiv durch den Therapeuten geschehen, um die Wahrnehmung des Patienten für die Neutralposition zu schulen ( $>$ Abb. 4), und in weiterer Folge über verschiedene Feedback-Variationen (Spiegel, Video etc.) assistiv oder aktiv geübt werden. Aufbauend auf dieser Fähigkeit, die Skapula in Neutralstellung zu halten, werden nun Bewegungen des Arms initiiert. Während der Armbewegungen versucht der Patient, die Neutralposition der Skapula kognitiv zu kontrollieren. Auch hier können dem Patienten unterschiedliche Hilfestellungen geboten werden, die einen effizienteren Lernerfolg gewährleisten, wie zum Beispiel taktiles oder visuelles Feedback ( $>$ Abb. 5). Bei Verwendung von Bracing Strategien und bei Verlust der Neutralposition wird neu begonnen. Als Progression wird schrittweise jegliches Feedback weggenommen.

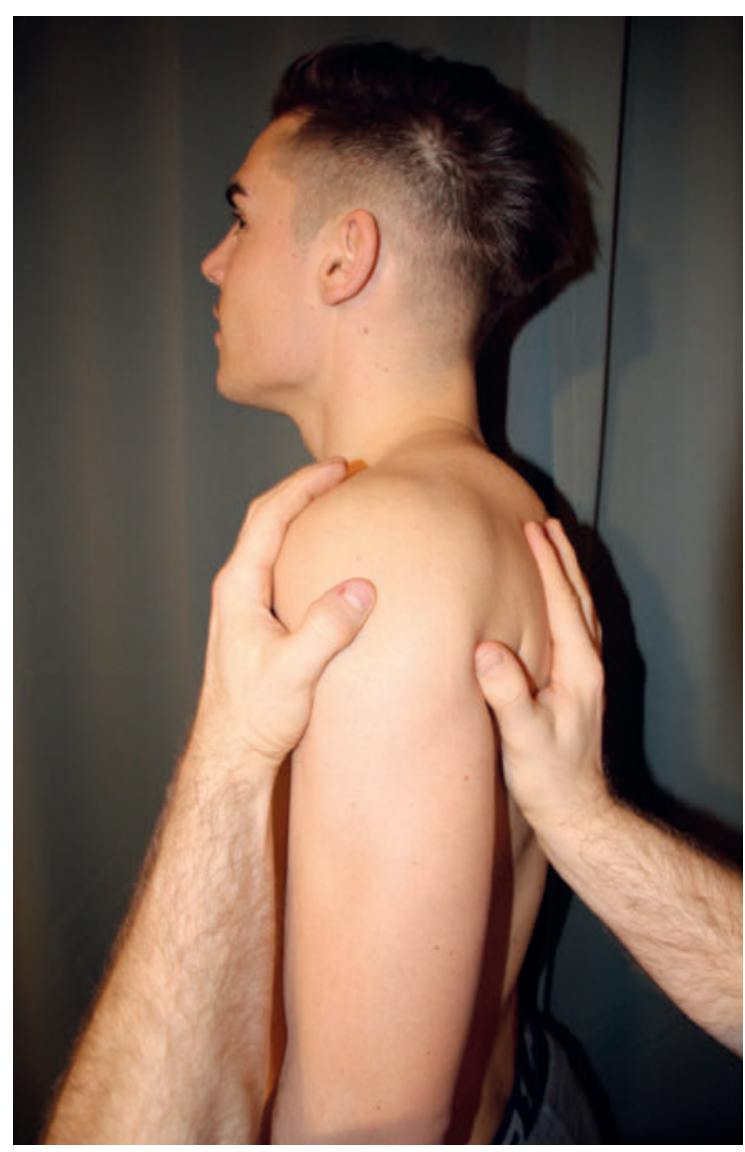

Abb. 4 Manuelle Korrektur der Skapula in die neutrale Trainingsposition (Symbolbild). (Quelle: T. Karlinger) 


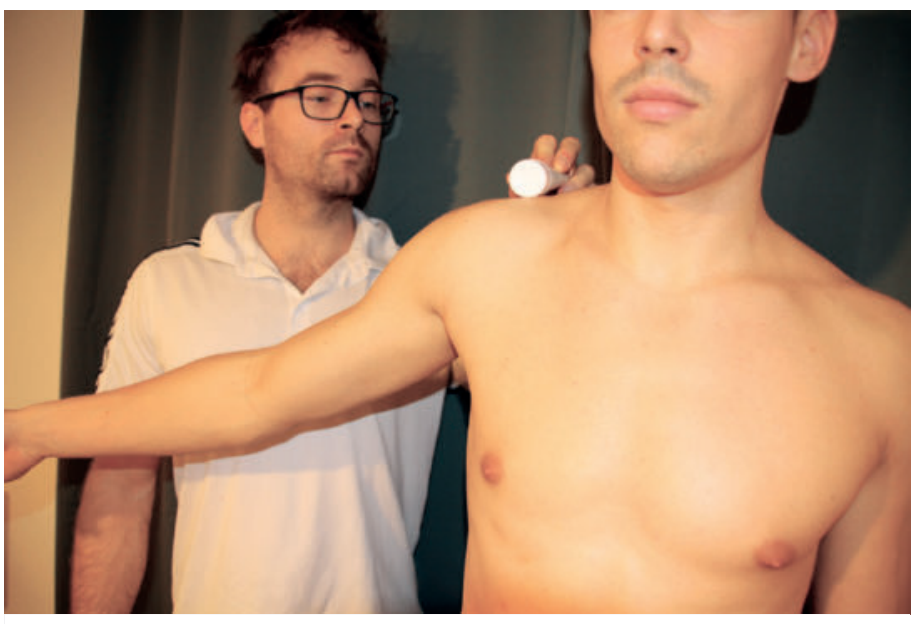

- Abb. 5 Retraining der Skapulakontrolle durch taktiles Feedback. Der Patient soll den Kontakt halten, während er den Arm in Abduktion bewegt (Symbolbild). (Quelle: T. Karlinger)

Das Retraining der Skapulakontrolle wird bei einem Trainingsvolumen von zwei Minuten, zweimal täglich, bei einem Bewegungstempo von drei Sekunden konzentrisch, drei Sekunden exzentrisch trainiert. Dies konnte das fehlerhafte Aktivierungsmuster von M. serratus anterior und M. trapezius pars ascendens bei Patienten mit Schulterimpingement wiederherstellen [9].

\section{Muskelspezifisches Retraining}

Die Wiederherstellung optimaler Bewegungsgesundheit der Skapula hört nicht mit dem Training der Koordination des Bewegungsmusters auf, sondern sollte auch muskelspezifisches Retraining miteinbeziehen.

Ein Schlüsselmuskel für die Skapulakontrolle ist der M. serratus anterior ( $\vee$ Abb. 6). Während dieser Muskel bisher oft als einzelner Muskel mit einer einzigen Funktion betrachtet wurde, haben neue funktionell anatomische Studien ergeben, dass sich der M. serratus anterior in einen oberen, mittleren und unteren Anteil unterteilen lässt ( $\mathbf{A} \mathbf{b b} \mathbf{b} \mathbf{6}$ ). Der obere Anteil, der von der ersten Rippe und dem oberen Teil der zweiten Rippe zum Angulus superior zieht, beeinflusst die Rotationsachse der Skapula und kontrolliert somit die Aufwärtsrotation. Der mittlere Anteil, der von dem unteren Teil der zweiten Rippe und der dritten Rippe zum Margo medialis scapulae zieht, besitzt die größte Cross sectional Area, produziert eine Außenrotation der Skapula mit anschließender Protraktion und kontrolliert damit das Winging. Der untere Anteil hat seinen Ursprung auf den Rippen vier bis acht/neun und setzt am Angulus inferior scapulae an. Dieser hat die Aufgabe, den Angulus inferior nach lateral um den Brustkorb zu ziehen. In Kombination mit den mittleren Fasern und dem M. trapezius pars ascendens kontrolliert dieser Muskelanteil wahrscheinlich den anterioren Tilt der Skapula [17].

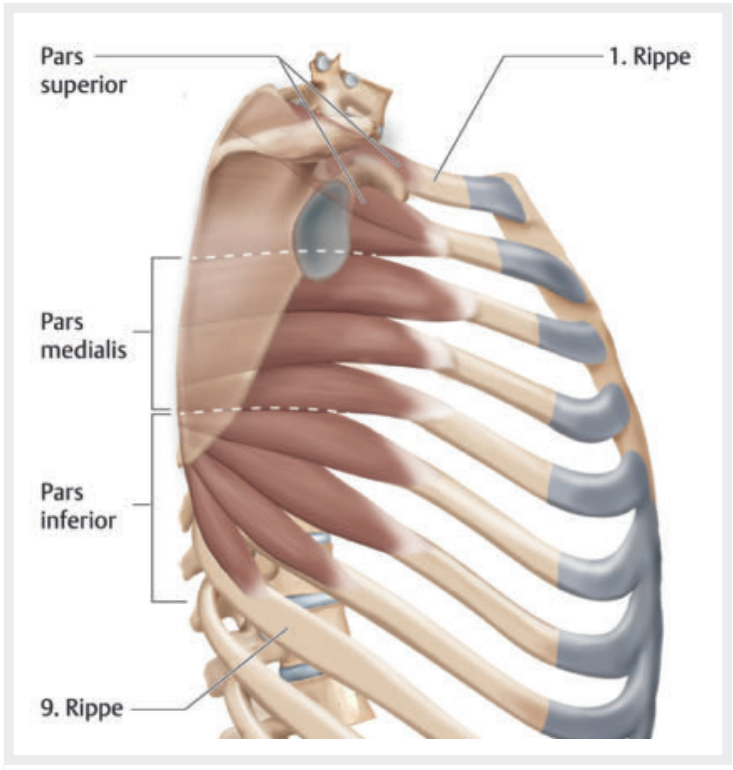

Abb. 6 M. serratus anterior. (Quelle: Hochschild J. Strukturen und Funktionen begreifen, Funktionelle Anatomie. Band 1: Wirbelsäule und obere Extremität. 5. Aufl. Stuttgart: Thieme; 2019)

Anhand dieser Einteilung wird der Beitrag dieses Muskels zur Bewegungsgesundheit der Skapula klar. Der M. serratus anterior bestimmt die Drehachse der Aufwärtsrotation, verhindert Skapula-Winging und zieht den Angulus inferior in Richtung Aufwärtsrotation. In Untersuchungen konnte wiederholt gezeigt werden, dass eine Einschränkung dieser Bewegungen der Skapula in Zusammenhang mit Nackenschmerzen und auch Schulterschmerzen stehen [18][19][20].

Diese Einteilung wirft jedoch auch die Frage auf, ob eine einfache „Push-Up-Plus“ Übung bis zum Bewegungsende der Protraktion ausreicht, um die Effizienz dieses komplexen Muskels wiederherzustellen. Untersuchungen wie diese bestätigen nur, dass es keine einfachen Antworten auf komplexe Fragen gibt. Unter Berücksichtigung dieser anatomischen Untersuchungen sollte beim muskelspezifischen Retraining des $\mathrm{M}$. serratus anterior darauf geachtet werden, dass die Aufwärtsrotation der Skapula (Akromion höher als Angulus superior) während jeglicher Art von Protraktionsübung erhalten bleibt.

\section{Fazit}

Bewegungsgesundheit bedeutet, dass dem Individuum viele Strategien zur Verfügung stehen, um eine bestimmte Bewegung durchzuführen. Klinisch untersucht wird die koordinative Fähigkeit, die Skapula in der Neutralposition zu halten, während der Arm in verschiedene Richtungen bewegt wird. 
Der Autor dankt Comera Movement Science (CMS), Sarah Mottram (Head of CMS) und Lincoln Blandford (Head of Education of CMS) für ihren Beitrag zu den theoretischen und praktischen Inhalten dieses Artikels.

\section{Autorinnen/Autoren}

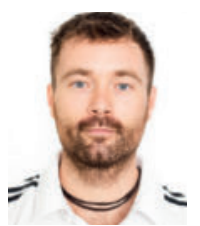

\section{Thomas Karlinger}

arbeitet als freiberuflicher MSK Physiotherapeut in Österreich. Neben seiner klinischen Tätigkeit unterrichtet er Kinetic Control Kurse im deutschsprachigen Raum.
Literaturverzeichnis am Ende der HTML-Version unter www.thieme-connect.de/products/manuelletherapie

\section{Bibliografie}

DOI https://doi.org/10.1055/a-1106-7833

manuelletherapie 2020; 24: 62-67

(c) Georg Thieme Verlag KG Stuttgart · New York ISSN 1433-2671

\section{Korrespondenzadresse}

\section{Thomas Karlinger}

thomas@perform-physio.at 